\title{
Classification by Type of the Arches in Gaudí's Palau Güell
}

\author{
Genaro González ${ }^{1} \cdot$ Albert Samper $^{2}$ • \\ Blas Herrera $^{3}$ (D)
}

\begin{abstract}
Architects and historians commonly claim that when designing the Palau Güell, Gaudí experimented with some architectural elements which would influence his later works, with the arches being prevalent in the building. But, to date, no mathematically thorough study has been conducted in order to determine the type of each of such arches. Furthermore, literature contains subjective and intuitive claims which create ambiguity and contradictions. Owing to the methods used by Gaudí, the arches in Palau Güell are best fit by conical curves (ellipse, parabola, hyperbola) or hyperbolic-cosine curves (catenary, Rankine). Using photogrammetrical and mathematical techniques, in this paper we will classify the arches of this building according to their types, we will eliminate the inconsistencies which currently exist in literature, and we will obtain the corresponding analytical equations, which may be helpful in future studies.
\end{abstract}

Keywords Palau Güell · Antoni Gaudí · Arch · Geometric analysis · Conics · Catenary · Rankine

Blas Herrera

blas.herrera@urv.cat

Genaro González

genaro.gonzalez@urv.cat

Albert Samper

albert.samper@urv.cat

1 Departament d'Enginyeria Mecànica, Universitat Rovira i Virgili, Tarragona, Spain

2 Escola Tècnica Superior d'Arquitectura, Universitat Rovira i Virgili, Reus, Spain

3 Departament d'Enginyeria Informàtica i Matemàtiques, Universitat Rovira i Virgili, Avinguda Països Catalans, 26, 43007 Tarragona, Spain 


\section{Introduction}

The Palau Güell (1885-1890) is amongst the first important projects by Antoni Gaudí (1852-1926), and the only new construction he was able to finish. The building was commissioned by the Barcelona businessman Eusebi Güell Bacigalupi, who gave the architect total freedom in design (González and Lacuesta 2013).

The three reasons which led us to make the thorough mathematical analysis presented here are: the ambiguity in the literature concerning the arches' geometry; the fact that there is no original document from Gaudí stating the particular types of arches, and thus there is a need to define the arches used; and the possible applications of the equations that define the arches.

With regard to the first of these reasons, many studies acknowledge that the design of this building influenced Gaudí's later architectural work. Antoni González writes, "...makes own contributions which form the basis of Gaudí's subsequently developed repertoire" (González 1990: 19, our trans.) and elsewhere states that "during conception and design, the architect introduced the formal, spatial and constructive repertoire which granted him universal recognition" (González 1993: 19, our trans.). Others claim that Gaudí experimented with different types of arches in the Palau Güell: “...for several years, the palace became a big experimental workshop" (González and Lacuesta 2013: 35-36, our trans.). However, the existing literature contains mathematically non-rigorous claims regarding the types of arches used. Rainer Zerbst writes, “...Gaudí used the parabolic arch design, which would recur in all his later works and eventually become a common element in his projects" (Zerbst 1988: 82), interested readers may also read the following reference (Billington 1985: 184-185). Antoni González and Raquel Lacuesta state, without providing a demonstration, that "Antoni Gaudí experimented with all types of arches in Palau Güell" (González and Lacuesta 2013: 88-89, our trans.); they say "all types of arches" but without defining those types. When examining the building's access arch, various authors (2000; Giralt-Miracle 2002; Granell 2002; Huerta 2006) propose several contradicting arc types (the contradictions between claims are not convincingly resolved). Likewise, many intuitive, subjective and arbitrary claims have been made regarding the overall geometry of the building arches, as, for instance, these two statements: “...the recurring use of the parabolic arch" and "The parabolic arch was a prevailing element..." (Lahuerta 1992: 105).

In short, while arcs are the most common geometric elements used throughout the Palau Güell, this fact has generated a subjective and intuitive debate with the purpose of ascertaining their types, and while many claims have been made, as the quotations above show, no final conclusion has been reached.

With regard to the second reason for this study, to fill the need to define the arches this paper intends to classify into types the 23 arches which can be found in the indoor and outdoor spaces of Palau Güell (Figs. 1, 2, 3, 4). This classification serves a dual purpose:

1. To check whether Gaudí really experimented with different arch types in Palau Güell, as stated in several studies. The classification will focus on conical curves (ellipse, parabola, hyperbola) and hyperbolic-cosine curves (catenary, 


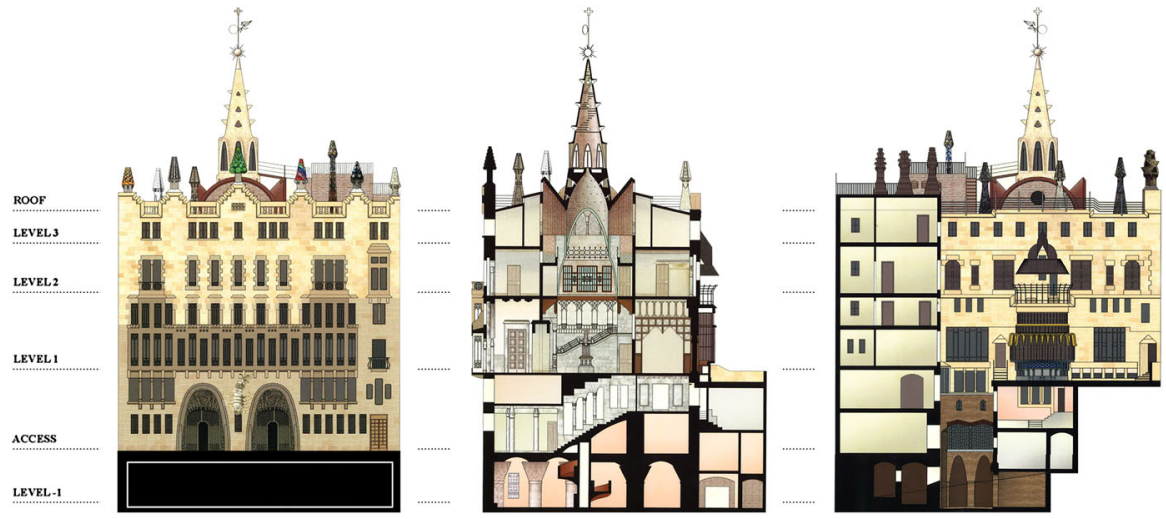

Fig. 1 On the left, main facade; in the center, section through the central dome of the building and on the right, section through the inner courtyard of the building. Arches 1-7 and 21 can be found on level -1 ; arches 18 and 20 can be found on entrance level; arches 8, 9, 15 and 16 can be found on level 1; arches 10 and 11 can be found on level 2; arches 12-14 and 19 can be found on level 3; arches 17, 22 and 23 can be found on the roof. Image: authors

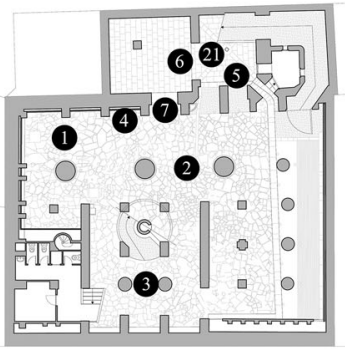

LEVEL -1

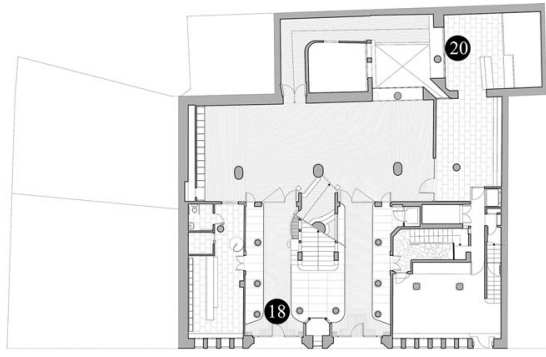

ACCESS

Fig. 2 Location of the arches on level -1 and entrance level of the building. This graphic document is reproduced with the permission of the Arxiu del Servei de Patrimoni Arquitectònic Local de la Diputació de Barcelona

Rankine), since these are the geometric shapes used by Gaudí to design his arches as we will show.

2. To eliminate the ambiguities and contradictions which, owing to the lack of thoroughness and mathematical objectivity, abound in studies and discussions regarding the type of each of the arches of the building.

Finally, with regard to the third reason for this present study, we will provide the normalized analytical equations of the best-fitting curves for all arches. These equations constitute additional information which may be useful for several reasons, including:

- In the field of architectural renovation, it is important to have an accurate knowledge of the object or space to be restored. Having geometric control of an object enables the technician to anticipate possible problems which might arise 


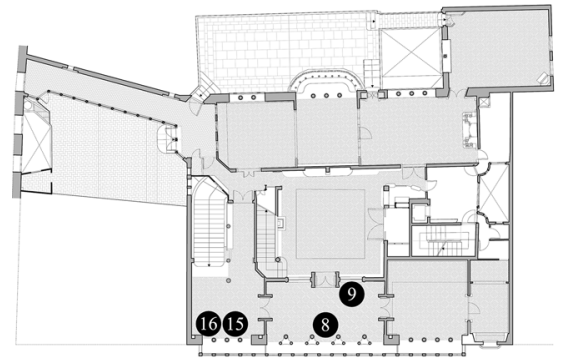

LEVEL 1

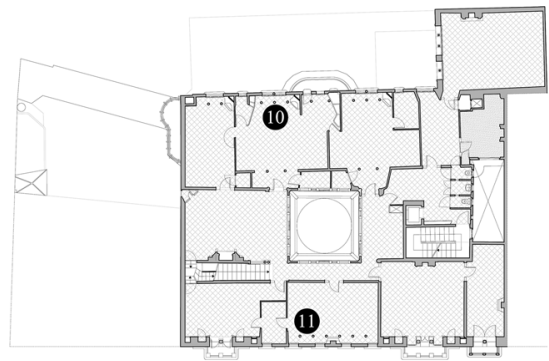

LEVEL 2

Fig. 3 Location of the arches on level 1 and level 2 of the building. This graphic document is reproduced with the permission of the Arxiu del Servei de Patrimoni Arquitectònic Local de la Diputació de Barcelona

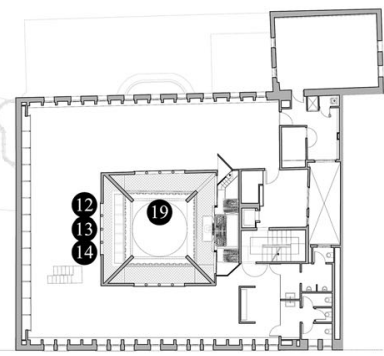

LEVEL 3

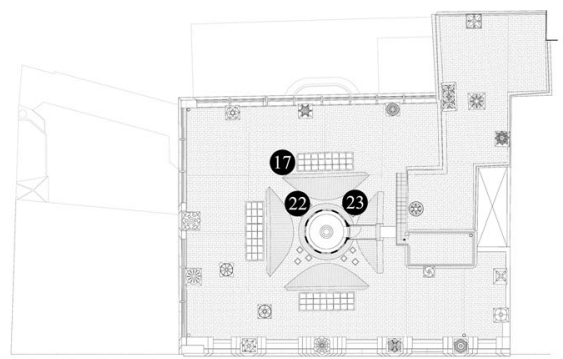

ROOF

Fig. 4 Location of the arches on level 3 and on the roof of the building. This graphic document is reproduced with the permission of the Arxiu del Servei de Patrimoni Arquitectònic Local de la Diputació de Barcelona

during reconstruction, or even to obtain an accurate estimate of the material costs and the amount of time needed to repair the arch at issue. The fact that Palau Güell has been listed as World Heritage by the UNESCO makes it important to know the precise geometry of each arch, should an intervention become necessary in the future.

- Having a detailed knowledge of the most representative elements of such an influential building by Gaudí may help to understand his work globally. The geometric analysis of the arches in Palau Güell may help to discover historical, formal and structural links with the arches used in other of his architectural works.

- In addition to the relevance for the field of architecture and architectural history, knowing the analytical equations can help to study the arches from other points of view. For instance, these equations could help monitor mechanical behaviour and come up with structural hypothesis for the arches; they could even help conduct a more detailed analysis of the acoustic impact on the spaces where the arches are located. Clearly, the arches play a strong role in many rooms of the Palau Güell. Besides, it is well known that parabolic and elliptical shapes direct 
the incident sound waves towards their respective focal points; therefore, the arches affect the acoustic comfort in spaces. Determining their geometries could help to globally control the acoustics and make specialized improvements.

In the next section, we briefly describe the geometric method used to determine the curve which best fits an architectural arch and its analytical equation, and then we apply this method to the 23 arches in Palau Güell.

\section{Brief Outline of the Method Used to Determine the Geometry of an Architectural Arch}

In this paper we use the method described in (Samper et al. 2017) to objectively determine which is the geometric shape within each of the conical curve types and hyperbolic-cosine curve types which best fits an arch of a heritage building. This method also provides an objective measurement of that fit, and does not involve mechanical, constructive or structural processes; it only involves standard geometric processes, numerical processes, computing, statistics and 3D data acquisition. Using this fitting method, for each type of conical curve (ellipse, hyperbola, parabola) or each type of hyperbolic-cosine curve (catenary, Rankine) we can determine which shape best fits a given arch, as well as the corresponding analytical equation and an objective measurement of that fit.

Interested readers may turn to (Samper et al. 2017) for a more detailed explanation of this method. What follows is only a brief description.

Our analysis starts with $\mathcal{N}^{\prime}=\left\{P_{i}^{\prime}\right\}_{i=1}^{i=n}$, which is the point cloud outlining the edge contour of an arch of Palau Güell. The points of this cloud were obtained using photogrammetrical techniques and the software PhotoScan (Fig. 5). For these points, we use $3 \mathrm{D}$ coordinates $\left(x^{\prime}, y^{\prime}, z^{\prime}\right)$ according to the $3 \mathrm{D}$ orthonormal coordinate system $C^{\prime}=\left\{p_{1} ; \vec{u}_{1}, \vec{u}_{2}, \vec{u}_{3}\right\}$ of the scanning device. It is to be noted that the spatial position of this reference $C^{\prime}$ is geometrically unknown at the start of calculations.

Next, the cloud $\mathcal{N}^{\prime}$ is orthogonally projected on plane $\sigma$, which is the architectural plane containing the edge of the arch. This projection renders a new bidimensional point cloud called $\mathcal{N}=\left\{P_{i}\right\}_{i=1}^{i=n}$. Using an orthonormal reference system $\mathcal{R}=\left\{P_{1}^{\prime} ; \vec{v}_{1}, \vec{v}_{2}\right\}$ which coordinates the points on $\sigma$ and is obtained using the Gram-Schmidt process, we calculate the conical regression. The resulting curve $\varepsilon \equiv B \bar{x}^{2}+C \bar{y}^{2}+D \bar{x} \bar{y}+E \bar{x}+F \bar{y}+1=0$ is the conical curve which best fits the projected point cloud, minimizing the sum of the quadratic residues $\sum_{i=1}^{i=n} \bar{\varepsilon}_{i}^{2}=\sum_{i=1}^{i=n}\left(B a_{i}^{2}+C b_{i}^{2}+D a_{i} b_{i}+E a_{i}+F b_{i}+1\right)^{2}$. The solution to the calculation problem of $\varepsilon$ is provided by the Gauss normal equations. Then, we consider another orthonormal reference system $C=\left\{\theta ; \vec{e}_{1}, \vec{e}_{2}\right\}$ for plane $\sigma$ made up of the following three geometric elements of the conical curve $\varepsilon$ in the system $\mathcal{R}$ : center $\equiv \theta=\left(\theta_{x}, \theta_{y}\right)$; major axis $\equiv(\bar{x}, \bar{y})=\theta+\lambda \vec{e}_{2}$ where $\lambda \in \mathbb{R}$; and minor axis $\equiv$ $(\bar{x}, \bar{y})=\theta+\lambda \vec{e}_{1}$ where $\lambda \in \mathbb{R}$. After all of the above, the points on $\sigma$ in the reference system $C$ have new coordinates $(\hat{x}, \hat{y})$. Therefore, $P_{i}=\left(\hat{x}_{i}, \hat{y}_{i}\right)$, where the 

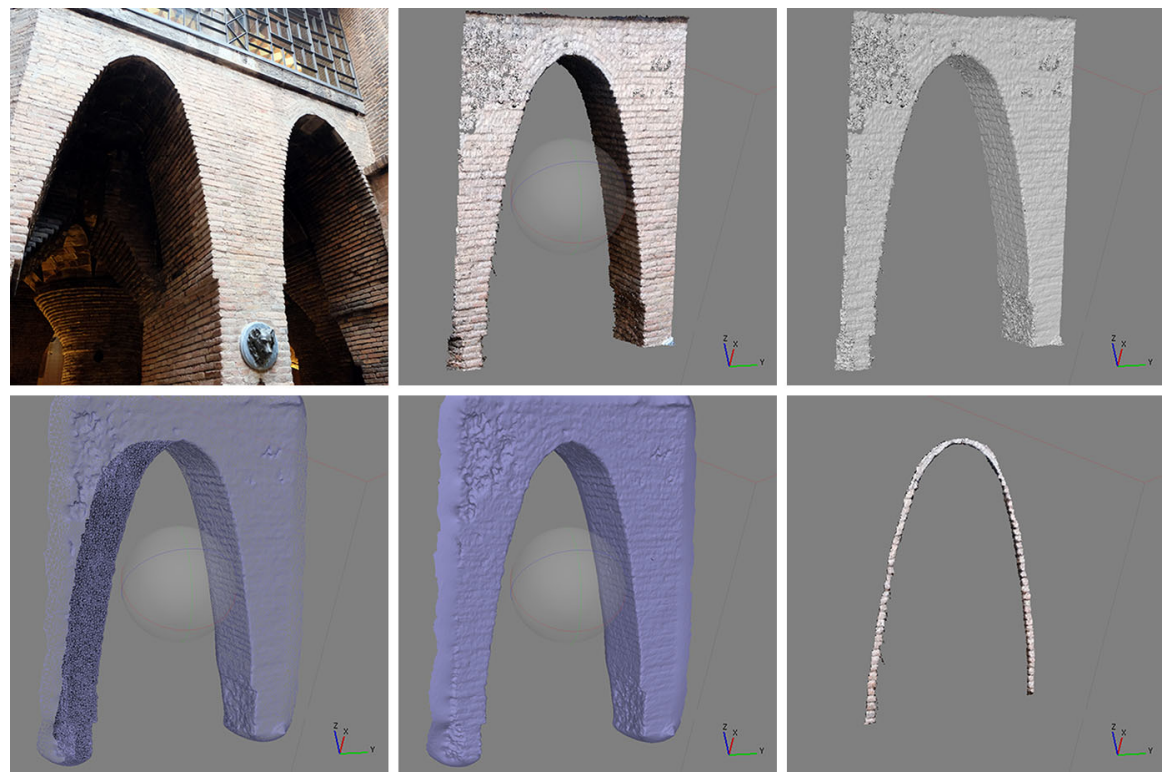

Fig. 5 Outline of the three-dimensional model of the arch 5 created with PhotoScan software. Image: authors

axis $\hat{Y}=\theta+\lambda \vec{e}_{2}$ is the central axis of the point cloud $\mathcal{N}$, as shown in (Samper et al. 2017).

Next we find a subset $\mathcal{B}=\left\{P_{i_{j}}=\left(\hat{x}_{i_{j}}, \hat{y}_{i_{j}}\right)\right\}_{i_{j}=1}^{i_{j}=100}$ of $\mathcal{N}$ using the algorithmic process described in (Samper et al. 2017). This subset determines the arch edge, and we call it edge cloud. Next we normalize the coordinates of the edge cloud $\mathcal{B}$. To that effect, we impose the condition that the lowest point in $\mathcal{B}$ must have coordinates $(1,0)$. This normalization is the equivalent of a change of coordinates to a new reference system $\mathcal{G}=\left\{v ; \vec{n}_{1}, \vec{n}_{2}\right\}$. The points on $\sigma$ in the system $\mathcal{G}$ have coordinates $(x, y)$, and the points of cloud $\mathcal{B}=\left\{P_{i_{j}}\right\}_{i_{j}=1}^{i_{j}=100}$ in the system $\mathcal{G}$ have coordinates $\left(x_{i_{j}}, y_{i_{j}}\right)$.

After defining the final reference system $\mathcal{G}$ (which, as we have seen, originates from the conical regression curve $\varepsilon$ as a result of geometric transformations of the cloud $\mathcal{N}^{\prime}$, and is therefore an intrinsic system of the cloud itself), we will find the conical regression curves and hyperbolic-cosine regression curves for the point cloud $\mathcal{B}$ of the arch's edge, obtaining their equations in the system $\mathcal{G}$. 
We calculate $\mathbf{P} \equiv y=A x^{2}+B$, the parabola regression curve of $\mathcal{B}$, and we obtain its equation in the reference system $\mathcal{G}$. This parabola regression curve is the parabola which best fits the point cloud $\mathcal{B}$, minimizing the sum of the quadratic residues $\sum_{i_{j}=1}^{i_{j}=100} \varepsilon_{p i_{j}}^{2}=\sum_{i_{j}=1}^{i_{j}=100}\left(A x_{i_{j}}^{2}+B-y_{i_{j}}\right)^{2}$. Its equation is given by the Gauss normal equations. These Gauss normal equations, as well as the rest of equations referring to the ellipse, hyperbola and hyperbolic cosine, as mentioned below, can be found in (Samper et al. 2017).

Similarly, we calculate $\mathbf{E} \equiv y=\frac{1}{2} F \pm \frac{1}{2} \sqrt{F^{2}+4 B x^{2}+4 G}, B<0$, the ellipse regression curve of $\mathcal{B}$, and we obtain its equation in the reference system $\mathcal{G}$. This ellipse regression curve is the ellipse which best fits the point cloud $\mathcal{B}$, minimizing the sum of the quadratic residues $\sum_{i_{j}=1}^{i_{j}=100} \varepsilon_{e i_{j}}^{2}=\sum_{i_{j}=1}^{i_{j}=100}\left(B^{\prime} x_{i_{j}}^{2}+C^{\prime} y_{i_{j}}^{2}+F^{\prime} y_{i_{j}}+1\right)^{2}, B^{\prime} C^{\prime}<0, \quad B=\frac{B^{\prime}}{-C^{\prime}}, \quad F=\frac{F}{-C^{\prime}}$, $G=\frac{1}{-C^{\prime}}$. As before, its equation is given by the Gauss normal equations.

We calculate $\mathbf{H} \equiv y=\frac{1}{2} F-\frac{1}{2} \sqrt{F^{2}+4 B x^{2}+4 G}, B>0$, the hyperbola regression curve of $\mathcal{B}$, and we obtain its equation in the reference system $\mathcal{G}$. This hyperbola regression curve is the hyperbola which best fits the point cloud $\mathcal{B}$, minimizing the sum of the quadratic residues $\sum_{i_{j}=1}^{i_{j}=100} \varepsilon_{h i_{j}}^{2}=\sum_{i_{j}=1}^{i_{j}=100}\left(B x_{i_{j}}^{2}-y_{i_{j}}^{2}+F y_{i_{j}}+G\right)^{2}, B>0$. The solution to this problem is not given by the Gauss normal equations, but by a critical point calculation. The method and the critical point equations which provide the solution can be found in (Samper et al. 2017).

After all of the above, we have determined which conical curves show the best regression fit to the cloud $B$. Now we continue with the following hyperbolic-cosine curves:

We calculate $\mathbf{C H} \equiv y=A \cosh (x)+B$, the hyperbolic cosine regression curve of $B$, and we obtain its equation in the reference system $\mathcal{G}$. This hyperbolic cosine regression curve is the hyperbolic cosine which best fits the point cloud $\mathcal{B}$, minimizing the sum of the quadratic residues $\sum_{i_{j}=1}^{i_{j}=100} \varepsilon_{h i_{j}}^{2}=\sum_{i_{j}=1}^{i_{j}=100}\left(A \cos \left(x_{i_{j}}\right)+B-y_{i_{j}}\right)^{2}$. As before, its equation is given by the Gauss normal equations. Hyperbolic cosine curves are a particular case of Rankine curves (discussed further below), but the corresponding numerical calculation is a preliminary step for the method used to calculate the two types of curves used by Gaudí in his projects: catenary curves and Rankine curves.

We calculate $\mathbf{C} \equiv y=A \cosh \left(\frac{x}{A}\right)+B$, the catenary regression curve of $\mathcal{B}$, and we obtain its equation in the reference system $\mathcal{G}$. This catenary regression curve is the catenary which best fits the point cloud $\mathcal{B}$, minimizing the sum of the quadratic residues $\sum_{i_{j}=1}^{i_{j}=100} \varepsilon_{c i_{j}}^{2}=\sum_{i_{j}=1}^{i_{j}=100}\left(A \cosh \left(\frac{x_{i_{j}}}{A}\right)+B-y_{i_{j}}\right)^{2}$. The solution to this problem is not given by the Gauss normal equations, but by a critical point calculation. The method and the critical point equations which provide the solution can be found in (Samper et al. 2017). 
Table 1 Analytical and typological results for the 23 arches in Palau Güell

\begin{tabular}{|c|c|c|c|}
\hline \# & Type & Equation & $P$ \\
\hline 1 & Circle & $y=A+\frac{1}{2} \sqrt{B-4 x^{2}}$ where $\mathrm{A} \approx-1.357, B \approx 11.263$ & 99.418 \\
\hline 2 & Circle & $y=A+\frac{1}{2} \sqrt{B-4 x^{2}}$ where $A \approx-1.156, B \approx 9.242$ & 99.879 \\
\hline 3 & Circle & $y=A+\frac{1}{2} \sqrt{B-4 x^{2}}$ where $A \approx-0.695, B \approx 5.720$ & 99.379 \\
\hline 4 & Parabola & $y=A x^{2}+B$ where $A \approx-1.313, B \approx 1.274$ & 99.815 \\
\hline 5 & Catenary & $y=A \cosh \left(\frac{x}{A}\right)+B$ where $A \approx-2.848, B \approx 2.943$ & 99.891 \\
\hline 6 & Ellipse & $y=A+\frac{1}{2} \sqrt{B+C x^{2}}$ where $A \approx-1.335, B \approx 15.925, C \approx-8.996$ & 99.939 \\
\hline 7 & Catenary & $y=A \cosh \left(\frac{x}{A}\right)+B$ where $A \approx-1.601, B \approx 1.598$ & 99.967 \\
\hline 8 & Ellipse & $\begin{array}{l}y=A+\frac{1}{2} \sqrt{B+C x^{2}} \\
\text { where } A \approx-7.642, B \approx 495.667, C \approx-272.207\end{array}$ & 99.813 \\
\hline 9 & Hyperbola & $\begin{array}{l}y=A-\frac{1}{2} \sqrt{B+C x^{2}} \\
\text { where } A \approx 126.007, B \approx 61229.180, C \approx 2227.418\end{array}$ & 99.956 \\
\hline 10 & Ellipse & $\begin{array}{l}y=A+\frac{1}{2} \sqrt{B+C x^{2}} \\
\text { where } A \approx-9.101, B \approx 453.016, C \approx-124.530\end{array}$ & 99.730 \\
\hline 11 & Ellipse & $y=A+\frac{1}{2} \sqrt{B+C x^{2}}$ where $A \approx-86.757, B \approx 31362.976, C \approx-1281.430$ & 99.934 \\
\hline 12 & Hyperbola & $\begin{array}{l}y=A-\frac{1}{2} \sqrt{B+C x^{2}} \\
\text { where } A \approx 15.215, B \approx 797.854, C \approx 134.436\end{array}$ & 99.765 \\
\hline 13 & Catenary & $\begin{array}{l}y=A \cosh \left(\frac{x}{A}\right)+B \\
\text { where } A \approx-1.906, B \approx 1.636\end{array}$ & 99.050 \\
\hline 14 & Parabola & $y=A x^{2}+B$ where $A \approx-1.317, B \approx 1.210$ & 99.769 \\
\hline 15 & Hyperbola & $\begin{array}{l}y=A-\frac{1}{2} \sqrt{B+C x^{2}} \\
\text { where } A \approx 15.271, B \approx 704.435, C \approx 258.785\end{array}$ & 99.503 \\
\hline 16 & Ellipse & $\begin{array}{l}y=A+\frac{1}{2} \sqrt{B+C x^{2}} \\
\text { where } A \approx-6.008, B \approx 295.810, C \approx-157.245\end{array}$ & 99.728 \\
\hline 17 & Circle & $y=A+\frac{1}{2} \sqrt{B-4 x^{2}}$ where $A \approx-0.445, B \approx 4.645$ & 99.682 \\
\hline 18 & Rankine & $y=A \cosh (C x)+B$ where $A \approx-0.033, B \approx 2.542, C \approx 5.101$ & 99.724 \\
\hline 19 & Ellipse & $y=A+\frac{1}{2} \sqrt{B+C x^{2}}$ where $A \approx-2.310, B \approx 58.971, C \approx-40.561$ & 99.598 \\
\hline 20 & Circle & $y=A+\frac{1}{2} \sqrt{B-4 x^{2}}$ where $A \approx-0,055, B \approx 3,896$ & 99.837 \\
\hline 21 & Circle & $y=A+\frac{1}{2} \sqrt{B-4 x^{2}}$ where $A \approx-2,552, B \approx 30,352$ & 99.546 \\
\hline 22 & Rankine & $y=A \cosh (C x)+B$ where $A \approx-0.816, B \approx 5.891, C \approx-2.727$ & 99.290 \\
\hline 23 & Catenary & $y=A \cosh \left(\frac{x}{A}\right)+B$ where $A \approx-2.958, B \approx 3.075$ & 99.171 \\
\hline
\end{tabular}

Finally, we calculate $\mathbf{R} \equiv y=A \cosh (C x)+B$, the Rankine regression curve of $\mathcal{B}$, and we obtain its equation in the reference system $\mathcal{G}$. The classic expression (Rankine 1877: 173-177; Merriman 1920: 723) of a Rankine curve is: $y=A \cosh \left(x \frac{2}{l} \operatorname{arccosh}\left(\frac{A+h}{A}\right)\right)+B$, where $l$ and $h$ are the clear span and the height, 


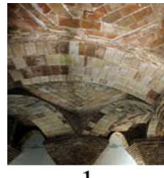

1

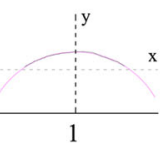

$\mathrm{L}=2 \mathrm{u}$

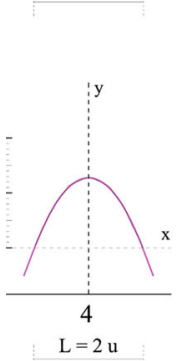

CIRCUMFERENCE

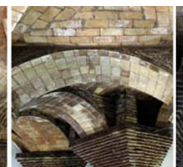

2

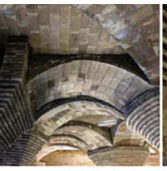

3

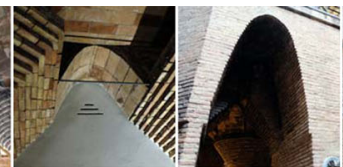

5

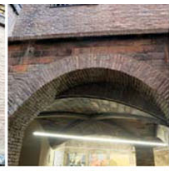

6

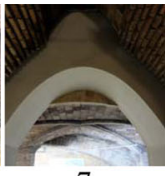

7
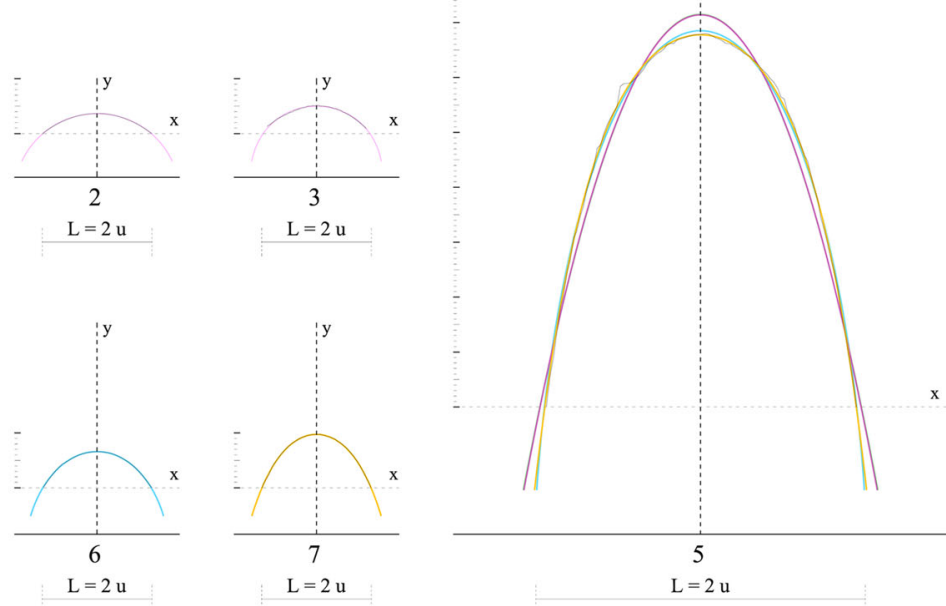

PARABOLA

ELLIPSE

HYPERBOLA

CATENARY

RANKINE

Fig. 6 Graphic results for arches 1-7. On the right, in a larger format, arch number 5 with the geometric regressions which best fit its original design. This arch is a case of coincidence between the best-fitting catenary curve and the best-fitting Rankine curve. Image: authors

respectively, of curve $\mathbf{R}$ on the reference system $\left\{V ; \vec{n}_{1},-\vec{n}_{2}\right\}$ where $V$ is the vertex of the curve.

We note that we do not call $\mathbf{R}$ a flattened catenary curve because it is not a catenary curve. Nor do we call it a general hyperbolic cosine curve, because it is not a hyperbolic cosine, but rather the analytical composition of a hyperbolic cosine with a homothetic transformation. We do not call it a funicular curve, because it does not correspond with the line obtained by hanging a finite number of weights from a chain.

This Rankine regression curve $\mathbf{R} \equiv y=A \cosh (C x)+B$ is the Rankine curve which best fits the point cloud $\mathcal{B}$, minimizing the sum of the quadratic residues $\sum_{i_{j}=1}^{i_{j}=100} \varepsilon_{r i_{j}}^{2}=\sum_{i_{j}=1}^{i_{j}=100}\left(A \cosh \left(C x_{i_{j}}\right)+B-y_{i_{j}}\right)^{2}$. The method and the critical point equations which provide the solution can be found in (Samper et al. 2017).

After all of the above, we have obtained the specific analytic equations of the normalized regression curves $\mathbf{P}, \mathbf{E}, \mathbf{H}, \mathbf{C}$ and $\mathbf{R}$ for the cloud $\mathcal{B}$ corresponding to the edge of an arch from Palau Güell.

Next we will calculate to what extent each of these curves statistically explains the cloud $\mathcal{B}$. For these calculations, we will use Pearson's adjusted coefficient of determination $\eta_{a d j}^{2}$, which is given by the equations described in (Samper et al. 2017). This coefficient $\eta_{a d j}^{2} \in[0,1]$ in all cases, and the value $P=\eta_{a d j}^{2} \times 100$ is the 


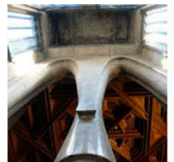

8
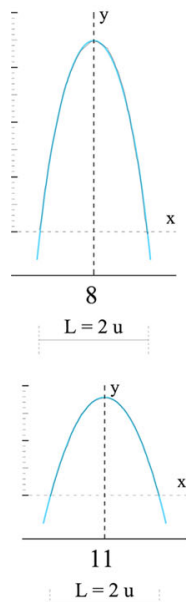

$\mathrm{L}=2$

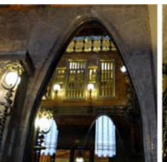

9
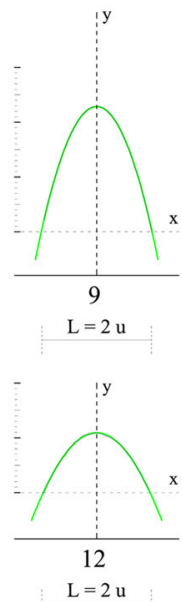

CIRCUMFERENCE

PARABOLA

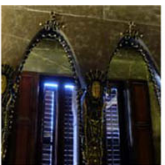

10

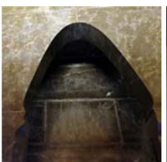

11

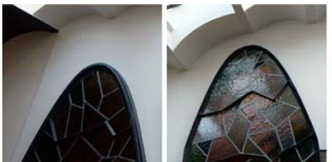

13

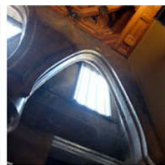

16
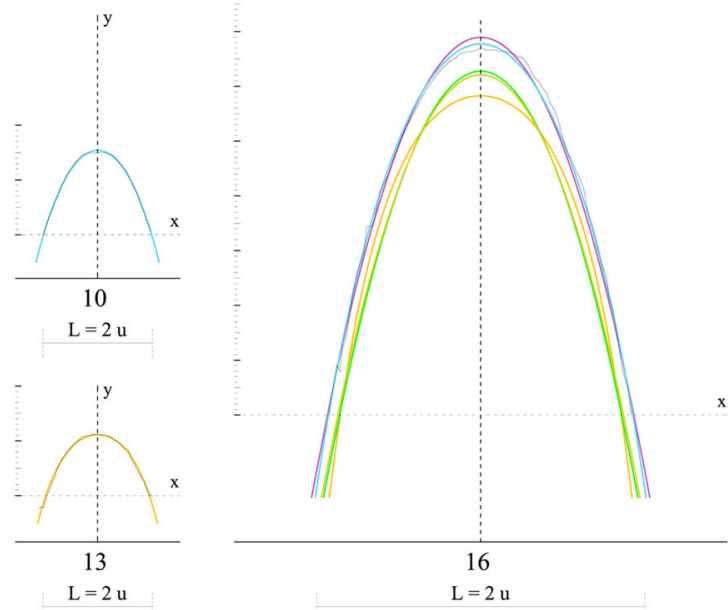

ELLIPSE

HYPERBOLA

CATENARY

RANKINE

Fig. 7 Graphic results for arches 8-13, and arch 16. On the right, in a larger format, arch number 16 with the geometric regressions which best fit its original design. Image: authors

proportion in which the variable $y_{i_{j}}$ of cloud $\mathcal{B}$ is statistically explained by the leastsquares correlation between $y_{i_{j}}$ and $x_{i_{j}}$. In other words, this value indicates the percentage of the variable $y_{i_{j}}$ of cloud $\mathcal{B}$ which is statistically explained by the corresponding regression curve.

If a much more in-depth geometric analysis is to be made, all the calculations can be repeated, not for the whole arch (that is, the whole cloud $\mathcal{B}$ ), but for different arch segments. This more in-depth geometric analysis can be found in (Samper et al. 2017). We have normalized the size of the arches, considering that an endpoint has coordinates $(1,0)$; again, an explanation of how the equations change if the scale changes can be found in (Samper et al. 2017).

\section{Results}

As stated before, our analysis starts with $\mathcal{N}^{\prime}=\left\{P_{i}^{\prime}\right\}_{i=1}^{i=n}$, which is the point cloud outlining the edge contour of an arch of Palau Güell. The points of this cloud were obtained using photogrammetrical techniques and the software PhotoScan. For these points, we use $3 \mathrm{D}$ coordinates $\left(x^{\prime}, y^{\prime}, z^{\prime}\right)$ according to the $3 \mathrm{D}$ orthonormal coordinate system $C^{\prime}=\left\{p_{1} ; \vec{u}_{1}, \vec{u}_{2}, \vec{u}_{3}\right\}$ of the scanning device. These initial coordinates are obtained to six decimal places, the results are presented to three decimal places. 


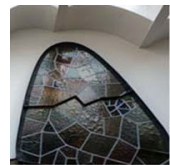

14

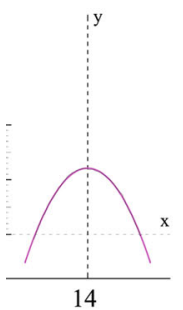

$\mathrm{L}=2 \mathrm{u}$

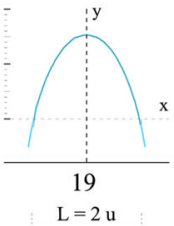

CIRCUMFERENCE

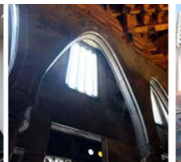

15

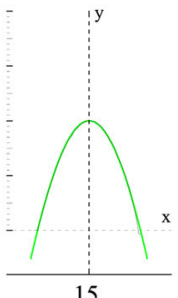

$\mathrm{L}=2 \mathrm{u}$

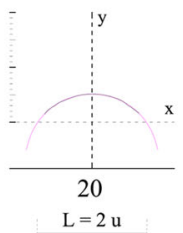

PARABOLA

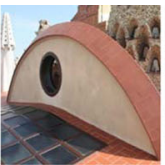

17

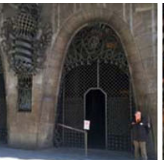

18

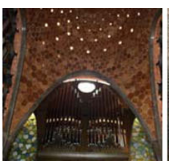

19

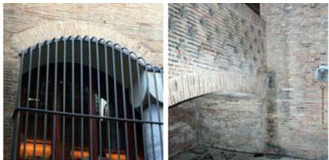

20

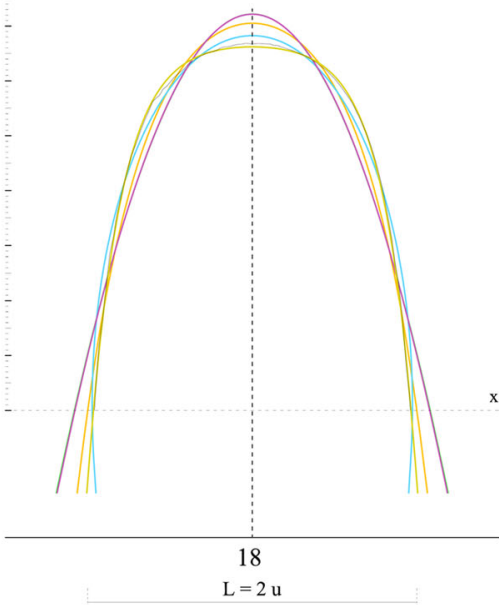

RANKINE

Fig. 8 Graphic results for arches 14, 15 and 17-21. On the right, in a larger format, arch number 18 with the geometric regressions which best fit its original design. Image: authors

After applying the method from (Samper et al. 2017), as mentioned in the previous section, the analytical and typological results for each arch are shown in Table 1 and, graphically, in Figs. 6, 7, 8 and 9.

The reader must take into account that:

- Even though the circle is a particular case of an ellipse, we consider them to be different cases.

- If the best-fitting curve for an arch is a catenary curve, then the best-fitting Rankine curve is the same as the best-fitting catenary curve for that arch.

\section{Conclusions}

Using standard geometric processes, numerical processes, computing, statistics and photogrammetrical data acquisition, we have classified the 23 arches from Palau Güell according to their geometrical types. The equations determined by us show that Gaudí indeed experimented with the four types of conic curves (circle, noncircular ellipse, parabola, hyperbola) and the two types of hyperbolic-cosine curves (Rankine, catenary). Hereinafter, those curves which are arcs of non-circular ellipses are called elliptical curves. 


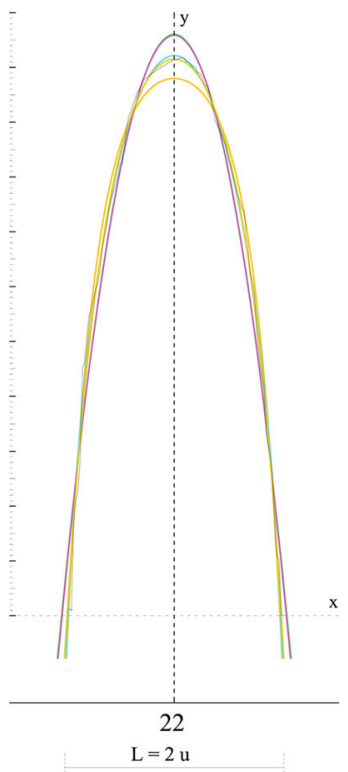

CIRCUMFERENCE

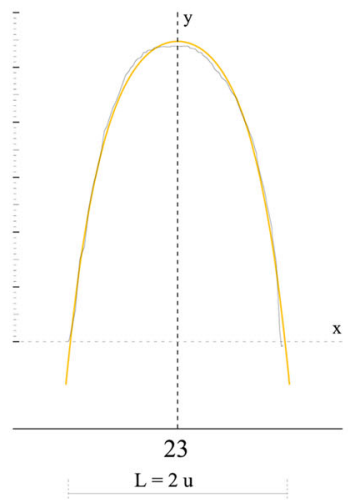

ELLIPSE

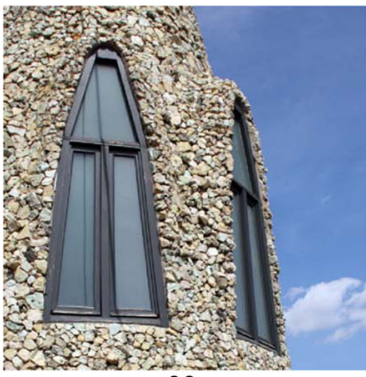

22

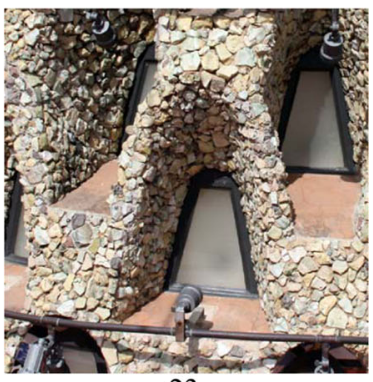

23

Fig. 9 Graphic results for arches 22 and 23. On the far left arch number 22 with the geometric regressions which best fit its original design. Image: authors

The arches are classified as follows (Table 1): $26.10 \%$ of them are best fit by a circular arc, $8.70 \%$ of them are best fit by a parabolic arc, $26.10 \%$ of them are best fit by an elliptical arc, $13.00 \%$ of them are best fit by a hyperbolic arc, $17.40 \%$ of them are best fit by a catenary arc, and $8.70 \%$ of them are best fit by a Rankine arc. Knowing this, we can make the following comments:

1. After searching the existing literature about Gaudi and his work, we have not found any rigorous geometric study concerning the use of elliptical arches. This is surprising given that $26.10 \%$ of the arches in Palau Güell are elliptical arches. Further, even though the curve type which best fits the access door of the building is a Rankine curve, the second best-fitting curve type (with a $\mathrm{P} \approx 97.82 \% \mathrm{fit}$ ) is an ellipse. This claim is made in (Samper et al. 2017), and can also be visualized in Fig. 5 of this present paper. However, as already said, it is surprising that this curve type is not mentioned in the existing literature.

2. Although in several pieces of specialized literature, such as (Zerbst 1988), it is claimed that the parabolic arch is the most frequently used arch in Palau Güell, it turns out that only $8.70 \%$ of the arches (that is, only 2 arches out of 23) are best fit by parabolic curves.

3. After analysing all the arches and their best-fitting curve types, it appears that Gaudí did not have any particular type of arch in mind for any particular architectural purpose (structural or ornamental function). There is no particular 
criterion with regard to the geometric type of the arches, with the sole exception of the arches which are best fit by circular curves, most of which are located in the lower areas of the building and clearly have a structural function.

4. Throughout history there are numerous examples of arches and historical buildings which were based on circular curves, elliptical curves, hyperboliccosine curves and parabolic curves (the latter shape resulting from an evenlyweighted hanging chain). The reason is twofold: firstly, the simple geometric tracing of these arches before actual construction, using common methods and tools; secondly, their geometric-structural properties. The case of the hyperbola is not as direct and intuitive, since the tracing is technically more complex. Despite that, in Palau Güell we find three hyperbolic arches.

Our analysis has allowed us to eliminate the ambiguities and contradictions that abound in studies and discussion regarding the type of each of the arches of the building.

The contents of this paper provide a preliminary step towards an analytical and geometric study regarding the behaviour of the categorized mathematical curves from the structural point of view and how they deform when they are subject to structural load. We believe it would be interesting to deepen the analysis of the relationship between surveyed shapes and structural behaviour, in order to highlight the connection between design intent and actual structural behaviour.

\section{References}

Billington, David P. 1985. The Tower and the Bridge: The New Art of Structural Engineering. Princeton: Princeton University Press.

Giralt-Miracle, Daniel (ed.). 2002. Gaudí, búsqueda de la forma: espacio, geometría, estructura y construcción. Barcelona: Lunwerg.

González, Antoni. 1990. The Palau Güell of Barcelona. The construction of a spatial idea. Informes de la Construcción 408 (42):17-22.

González, Antoni. 1993. Método y criterios en la restauración del Palau Güell de Barcelona. Informes de la Construcción 428, 45:19-38.

González, Antoni and Lacuesta, Raquel. 2013. El Palacio Güell: Una obra maestra de Antoni Gaudí. Barcelona: Diputació de Barcelona.

González, J. Luis. 2000. La organización constructiva del descenso de cargas del Palau Güell de Barcelona, obra de Antoni Gaudí. Actas del Tercer Congreso Nacional de Historia de la Construcción, Vol I: 443-450. Sevilla: Instituto Juan de Herrera.

Granell, Enrique. 2002. El Palau Güell de Antoni Gaudí en Barcelona: 1886-1888. DC PAPERS, revista de crítica y teoría de la arquitectura 7: 48-55.

Huerta, Santiago. 2006. Structural design in the work of Gaudí. Architectural Science Review 49(4): 324-339.

Lahuerta, Juan. 1992. Antoni Gaudí: 1852-1926. Arquitectura, ideología y política. Madrid: Electra.

Merriman, Mansfield. 1920. American civil engineers' handbook. New York: Wiley \& Sons.

Rankine, William J. Macquorn. 1877. A manual of applied mechanics. London: Griffin.

Samper, Albert, Genaro González and Blas Herrera. 2017. Determination of the geometric shape which best fits an architectural arch within each of the conical curve types and hyperbolic-cosine curve types: the case of Palau Güell by Antoni Gaudí. Journal of Cultural Heritage 25: 56-64.

Zerbst, Rainer. 1988. Antonio Gaudi. Cologne: Taschen. 
Genaro González is an architect who obtained his PhD in Architecture at the University Rovira i Virgili of Tarragona in 2015. Presently he is a full professor of mechanical engineering at the same university and his main fields of interest are mechanical engineering and the application of geometry to architecture.

Albert Samper is an architect who obtained his $\mathrm{PhD}$ in Architecture at the University Rovira i Virgili of Tarragona in 2014. Presently he is an associate of Architecture at the same university and his main fields of interest are fractal geometry and the application of geometry to architecture.

Blas Herrera is a geometer who obtained his DSc in Mathematics at the University Autonoma of Barcelona in 1994. Presently, he is a full professor of applied mathematics at the University Rovira $\mathrm{i}$ Virgili of Tarragona. His main fields of research interest are classical and differential geometry, and the application of geometry to architecture, fluid mechanics and engineering. 